\title{
Prospective protochordate homologs of vertebrate midbrain and MHB, with some thoughts on MHB origins
}

\author{
Thurston C. Lacalli \\ Biology Department, University of Victoria, Victoria, B.C., Canada, V8W-3N5. \\ Corresponding address: Thurston C. Lacalli, Tel: 1-(250) 598-9859. Fax: 1-(250) 721-7120. E-mail: lacalli@uvic.ca
}

Received: 2006.01.15; Accepted: 2006.04.01; Published: 2006.05.05

The MHB (midbrain-hindbrain boundary) is a key organizing center in the vertebrate brain characterized by
highly conserved patterns of gene expression. The evidence for an MHB homolog in protochordates is
equivocal, the "neck" region immediately caudal to the sensory vesicle in ascidian larvae being the best accepted
candidate. It is argued here that similarities in expression patterns between the MHB and the ascidian neck
region are more likely due to the latter being the principal source of neurons in the adult brain, and hence where
all the genes involved in patterning the latter will necessarily be expressed. The contrast with amphioxus is
exemplified by pax $2 / 5 / 8$, expressed in the neck region in ascidian larvae, but more caudally, along much of the
nerve cord in amphioxus. The zone of expression in each case corresponds with that part of the nerve cord
ultimately responsible for innervating the adult body, which suggests the spatially restricted MHB-like
expression pattern in ascidians is secondarily reduced from a condition more like that in amphioxus. Patterns
resembling those of the vertebrate MHB are nevertheless found elsewhere among metazoans. This suggests that,
irrespective of its modern function, the MHB marks the site of an organizing center of considerable antiquity.
Any explanation for how such a center became incorporated into the chordate brain must take account of the
dorsoventral inversion chordates have experienced relative to other metazoans. Especially relevant here is a
concept developed by Claus Nielsen, in which the brain is derived from a neural center located behind the
ancestral mouth. While this is somewhat counterintuitive, it accords well with emerging molecular data.

Key words: protochordates, midbrain/hindbrain boundary, brain evolution, ascidians, amphioxus, dorsoventral inversion

\section{Midbrains and MHBs in protochordates}

Molecular and morphological evidence together support the idea that the anterior end of the amphioxus nerve cord contains regions homologous with vertebrate forebrain and hindbrain [1,2], though it is generally the ventral portion of these regions that are best represented [3]. The presence of a midbrain homolog is more controversial. Its most probable position, as defined by gene expression patterns, would be from somewhere forward of the caudal limit of otx expression, which in vertebrates extends through the midbrain, to a point close to the beginning of the zone of Hox gene expression. In young amphioxus larvae, this corresponds with a region extending from the infundibular cells, which lie at a level roughly midway along somite 1 , through the posterior part of the cerebral vesicle to a level somewhere near or just beyond the middle of somite 2 (Fig. 1A). This domain begins with an anterior zone of ventral neuropile and commissural fibers followed, near the junction between somites 1 and 2 , by a complex of ventrally-positioned somatic motoneurons and interneurons with caudal projections that initiate and control larval swimming behavior [4]. The first members of the visceral motoneuron series lie further back, near the caudal end of somite 2. From there, visceral motoneurons recur at regular intervals along the anterior nerve cord and innervate the body via an extended series of peripheral nerves.

Any attempt to identify an amphioxus homolog of the vertebrate midbrain is hampered by the fact that we currently lack unambiguous criteria for recognizing its presence. The quintessential identifying feature in vertebrates is the dorsal optic tectum, but this is absent in amphioxus. In fact, except for a pineal homolog, amphioxus appears to lack all of the dorsal structures of the vertebrate brain. Further, because the amphioxus nerve cord is of uniform dimension along its length, there are no morphological constrictions to separate sub-domains in the anterior cord from one another in the way vertebrate isthmus separates midbrain from hindbrain. The isthmus is notable as the site of an important organizing center, the midbrain-hindbrain boundary (MHB), characterized in vertebrates by the expression of a highly conserved set of gene, including $f g f 8$, engrailed, pax 2 , and wnt1 $[5,6]$. The comparable site in amphioxus lies somewhere close to the caudal limit of somite 1 , which is where the anterior zone of otx expression abuts that of $g b x$ [7]. However, though engrailed is expressed in small clusters of cells in the embryonic nerve cord [8], these lie further forward in the cerebral vesicle, at a level near the midpoint of somite 1 . In addition, wnt1 is not expressed at all in the anterior cord, nor does the 
expression of the amphioxus pax2 homolog, AmphiPax $2 / 5 / 8$, match the vertebrate pattern. Instead, the latter is expressed caudally through much of the nerve cord [9], and though there is a small anterior zone of later expression, it is too far forward for an MHB marker.

Figure 1. Dorsal views of the larval nerve cords of (A) amphioxus and (B) Ciona, a representative ascidian, showing expression domains for the main CNS patterning genes. This is somewhat simplified, as the exact extent of the expression domains can vary with developmental stage, and the ascidian hox genes, in particular, are expressed in non-overlapping domains with some gaps.

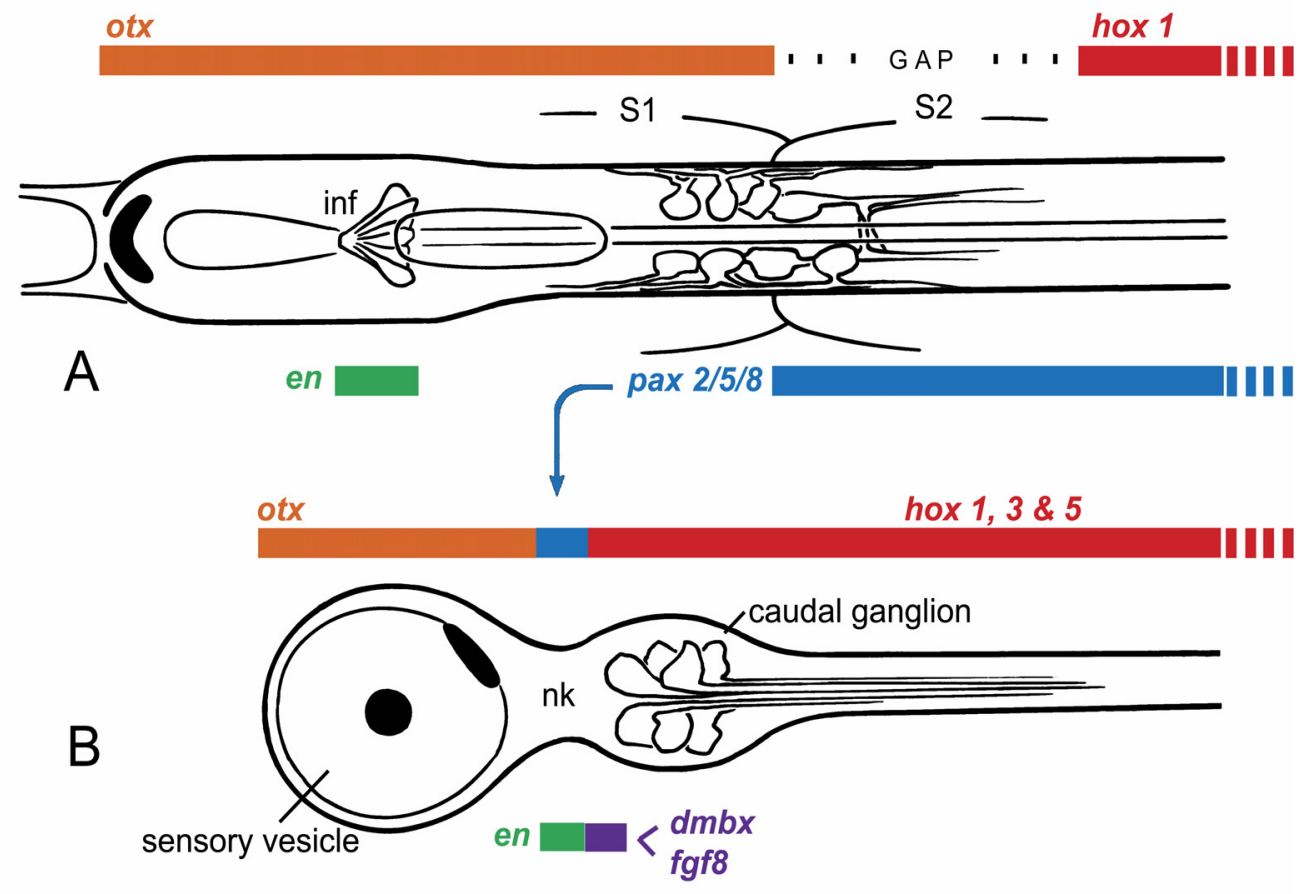

There is thus no clear molecular evidence for a focal center in amphioxus with the expression profile of an MHB. This is perhaps not surprising, considering that the structures organized by the $\mathrm{MHB}$ are primarily dorsal ones not present in amphioxus. The MHB is, however, also required for normal organization of some ventral brain regions in vertebrates [10]. Since the ventral midbrain of vertebrates very likely combines vertebrate-specific neuronal cell types along with cell groupings with more primitive organizational features, it would be useful to know whether all of these are MHBdependent or only the former. It may well be that only vertebrate innovations, whether dorsal or ventral, are under the specific control of the MHB. This leaves open the question of whether the amphioxus pattern differs from that of vertebrates because it reflects an earlier stage in the evolution of a vertebrate-type MHB [7], or whether amphioxus has secondarily diverged from a pattern that was once closer to the vertebrate one. In addition, the vertebrate midbrain marker $d m b x$ is not expressed in the amphioxus nerve cord [11]. The available molecular data thus argues against the presence, in amphioxus, of precise homologs of either the vertebrate midbrain or MHB.

Among tunicates, the other group of protochordates, it is the ascidians that are best studied, and for these there is again good molecular evidence for homologs of both forebrain and hindbrain in the larval CNS [11,12], corresponding roughly with the sensory vesicle and caudal ganglion respectively. In addition, cells in the narrow "neck" region that separates these structures express at least some characteristic MHB genes (Fig. 1B), notably pax $2 / 5 / 8$, engrailed, and $f g f 8[13,14]$. Data on pax $2 / 5 / 8$ led initially to the proposal that ascidians had an exact counterpart of the vertebrate $\mathrm{MHB}$ and that amphioxus, being a later offshoot of the chordate lineage, must have lost this feature secondarily [12]. However, the precise spatial arrangement of the expression zones for several key genes in ascidians differs from that in vertebrates, e.g. $f_{g} f$ and $d m b x$ in ascidian larvae are expressed in cells immediately caudal to those expressing pax2/5/8 and en, whereas in vertebrates, $d m b x$ lies forward of the other three 
genes, whose expression overlaps. Expression patterns of the same genes in larvaceans, another group of tunicates, is somewhat different yet again [13], which further complicates the problem of determining the nature of the ancestral pattern.

The molecular data is thus somewhat inconclusive regarding protochordate homologs of either the vertebrate midbrain or MHB. If anatomical and functional considerations are taken into consideration, however, a somewhat stronger case can be made that amphioxus may have an approximate counterpart of the midbrain. The key point is that some of the cell types and neural circuits located in the caudal part of the zone of otx expression are similar to those found in the ventral midbrain in vertebrates. In amphioxus, as pointed out above, only ventral markers are available for comparison. Of these, the infundibular cells probably mark the anterior limit of any prospective midbrain-like territory, as their homology with the ventral infundibular region of the vertebrate diencephalon seems to be fairly well accepted. Immediately behind this point, in amphioxus, there is a zone of ventral neuropile in some ways comparable with the ventral tegmental commissure, which forms part of the early axonal scaffolding in embryonic vertebrate brains. This same region in amphioxus also contains the anterior-most motoneurons in the nerve cord along with populations of large interneurons with descending projections, features found in the tegmentum and the reticulospinal plexus of lower vertebrates beginning at midbrain level. The ventral midbrain in vertebrates is also where dopaminergic neurons with projections to the forebrain first develop, and these are a key component of the motivational circuitry linking basal brainstem centers with the forebrain. Dopaminergic neurons are found in the cerebral vesicle in amphioxus and nowhere else in the nerve cord. Two main populations develop, one in a dorsal position in the anterior cerebral vesicle, the other more ventrally near the junction between somites 1 and $2[14,15]$. Further research is needed on these cells to determine their precise pattern of projections and function, but the more caudal of the two populations is well positioned to be a homolog of the midbrain dopaminergic neurons in vertebrates.

If the anatomical and functional criteria outlined above are meaningful indicators that amphioxus does indeed have a midbrain homolog, the value of the molecular markers used to date to test this would have to be reconsidered. The same could be said of ascidian larvae, but for a different reason, for here there is an alternative explanation for the expression patterns observed. The CNS of adult ascidians consists of a simple brain-like dorsal ganglion, from which nerves radiate to the body musculature and visceral organs. The ganglion is present in only a rudimentary form in the larva, however, and the source of its cells has never been clear. In the few species where this has been investigated in the past, the ganglion develops in contact with the neck region of the larval nerve cord near the site where it contacts the neurohypophyseal duct. The latter is partly a stomodeal derivative, which raises the question of whether a significant part of the ganglion might be derived from stomodeal ectoderm. An analysis of the salp ganglion, compared with the data available on compound ascidians [16,17], supports the idea that most if not all of the dorsal ganglion is of neural origin and that, in ascidians, it develops as part of the neck region. Recent data on Ciona [18] tends to support this interpretation, as its ganglion develops in contact with the base of an outgrowth that arises from the caudal part of the sensory vesicle very near the neck. More importantly, experimental work now in progress on Ciona is confirming the neck region as the source of major classes of neurons within the adult ganglion, including motoneurons innervating the visceral organs [J.F. Brunet, personal communication].

Evidently then, the cells of the neck region in ascidian larvae serve as a pool of precursors from which most if not all post-metamorphic CNS neurons are derived. Assuming the genes expressed in the vertebrate MHB include major players in the overall process of neuronal specification and differentiation, one can argue that their expression in the neck region is to be expected, irrespective of any homology between this site and the MHB. One would predict the genes would be expressed in combinatorial patterns and in a few cells at most, which is precisely what is observed.

Current thinking on the nature of ancestral chordates favors the view that the separation of adult and larval tissues in ascidians is a secondary specialization, and that the ancestral body plan was a more fully integrated one, as in amphioxus $[19,20]$. This may explain why the expression patterns of some key developmental control genes differ so dramatically between ascidians and amphioxus. Consider pax $2 / 5 / 8$, which has an extended zone of expression in amphioxus, but a very restricted one in ascidians (cf. Figs. 1A, B). Paralleling these, there are differences in innervation patterns of, for example, the visceral organs. These are strictly adult structures in ascidians, and the cells responsible for their innervation arise from the neck region, whereas the Hox-expressing part of the nerve cord, which innervates the tail, is entirely lost at metamorphosis. In amphioxus, in contrast, visceral motoneurons, along with the rest of the locomotory control system, arise from an extended region of the nerve cord extending well into the Hox zone. Whether the zone supplying visceral innervation corresponds precisely with that expressing pax2/5/8 has yet to be determined. One can nevertheless predict that any gene essential to the development of the visceral innervation will necessarily be expressed over a much greater length of the nerve cord in amphioxus than in ascidians. For at least some of the genes associated with the MHB, therefore, the reason their homologs have a very restricted expression zone in ascidian larvae likely has less to do with the presence of a 
vertebrate-type MHB, and more with the functional necessity of generating a full complement of adult neurons from a single site within the nerve cord. What one then wants to know, to determine whether the ascidian pattern and the vertebrate one are more than coincidentally related, is what functional role the genes play in each instance. With regard to pax $2 / 5 / 8$ specifically, a further clue might come from knowing whether its expression in hemichordate embryos is restricted, as in ascidians, or extended, as in amphioxus. If the latter, this would be further evidence for the view that the ascidian pattern is indeed the derived one.

\section{The ancestral condition - did it include a precursor of the MHB?}

From the above it is clear that there is a certain plasticity in the way expression patterns of genes identified with the MHB have diverged among chordates. In fact, the vertebrates seem to be most conservative: it is their pattern that most closely resembles that of hemichordates $[19,20]$, the closest available model for an ancestral deuterostome, and there are remarkably similar patterns in protostomes, e.g. Drosophila. In hemichordates it is the ectoderm that is patterned, since there is no internalized CNS. An MHB-like region can nevertheless be recognized, consisting of overlapping zones of en, pax $2 / 5 / 8, f g f 8$ and wnt 1 expression near the junction between the collar and trunk in a region where otx and $g b x$ expression overlaps [21]. If, however, as argued above, the specific organizer functions of the vertebrate $\mathrm{MHB}$ evolved with vertebrates, why are hemichordate expression patterns so close to vertebrate ones? It could be, that despite the anatomical differences in their nervous systems, homology dictates the position along the body axis at which particular neural functions and cell types are localized. This might be due, for example, to their association with conserved structures like the mouth or the first gill slit. Too little is currently known of hemichordate neuroanatomy to speculate much further, though some essential aspect of ectodermal and/or neural patterning is presumably involved. A further problem is to discover why amphioxus and ascidians have altered their MHB-related expression patterns when they are elsewhere so well conserved. In the case of ascidians, there have evidently been major changes in the way key patterning genes, notably those of the Hox cluster, are structured and regulated. This probably relates to the presence of alternative mechanisms for cell specification in ascidians, which has relaxed some constraints on genome organization [22]. It is otherwise somewhat surprising that such comparatively distant relatives as vertebrates and hemichordates are so similar in terms of their MHB-related expression patterns when the main protochordate groups are both so different from vertebrates and from each other.

There is a second issue integral to any consideration of chordate CNS origins that relates to how the main organ systems of the body, including the nervous system, are positioned in relation to the dorsoventral axis. Comparatively strong evidence now supports the idea that the body of chordates is dorsoventrally inverted relative to that of insects and the other main groups of protostomes. A full account of how this might have happened has been difficult to piece together, at least until now, because there was little relevant data on the basal offshoots of the lineage leading to chordates, namely hemichordates and echinoderms. Recent data from hemichordates has proved informative, however. Their anteroposterior maps are very similar to vertebrates [21] and, while the data on dorsoventral patterning is more ambiguous, it appears that hemichordates are oriented the same way as protostomes, such that the mouth is ventral and the expression domain for homologs of the dorsoventral patterning genes $b m p 2 / 4$ and $b m p 7$ is dorsal [20]. Removal of all influence of the latter in hemichordates results in a much expanded mouth, while over-expression suppresses the mouth and generates radialized embryos. In vertebrates, Bmp expression and the mouth are both ventral, but suppressing Bmps has equally dramatic radializing effects [23]. The genes thus appear to be performing essentially similar functions in these two groups, which implies that dorsal in basal deuterostomes has become ventral in vertebrates and, hence, in all chordates.

Inversion must therefore have occurred between the separation of the clade comprising hemichordates plus echinoderms from chordates, possibly in an organism that did not yet have an internalized CNS. The transition is somewhat easier to imagine, in fact, if the starting point is an animal with circumferential expression zones of all the key patterning genes (Fig. $2 \mathrm{~A})$. It should then be equally easy to concentrate both the expression zones and the structures they pattern either to the dorsal surface or the ventral one (Fig. 2C). Walter Garstang's ideas about chordate origins are fairly typical of hypotheses that predate the renewed interest in dorsoventral inversion. He derived neural tube from the dorsal portion of an ancestral system of larval ciliary bands, in that way preserving the dorsoventral orientation of the ancestor (Fig. 2B). If inversion did, in fact, occur, the brain and nerve cord would have had to come instead from the ventral surface of the ancestral body (Fig. 2C), an alternative explored by Claus Nielsen in a 1999 analysis [24]. A major premise of Garstang's hypothesis, that the whole of the neural tube should to arise as a single entity, is no longer widely accepted [19], but it is still useful to consider the consequence of dorsoventrally inverting his hypothetical ancestor. As Nielsen points out, so long as the brain arises at the anterior end of an originally ventral neuraxis, it must necessarily form in the postoral region, most likely immediately caudal to the mouth. This may seem counterintuitive, but the zone of expression for MHB-type genes, which mark a core brain region in vertebrates, is also postoral in hemichordates. 
Figure 2. Two alternatives for explaining the origin of a dorsally positioned brain in chordates, using the hemichordate embryo (A) as a starting point; indicates the mouth (m), anus (a), apical plate (orange), and the ancestral dorsal (a-dorsal) and ventral (a-ventral) surfaces; the light blue rectangle is the expression "hot zone" (hz) in which homologs of genes characteristic of the vertebrate MHB are expressed, typically in circumferential bands within ectoderm of the collar and anterior trunk. Older explanations of chordate origins, e.g. Garstang's hypothesis, retain an ancestral dorsoventral orientation (as in B). This allows the dorsal condensation of tissue that became the brain (dark blue) to form within the hot zone of MHB-like expression, co-opting the latter for neural patterning functions. With inversion (C, left side), the ancestral ventral surface becomes dorsal. In contrast with $\mathrm{B}$, any brain-like condensation that forms on the new dorsal surface would necessarily then be post-oral in position, but it could still incorporate the MHB-like hot zone. Then, to generate the vertebrate condition ( $\mathrm{C}$, right side), with mouth and brain on opposing sides of the body, one needs to move the mouth over the front or side of the snout by differential growth of an expansion zone (ez) located somewhere forward of the brain.
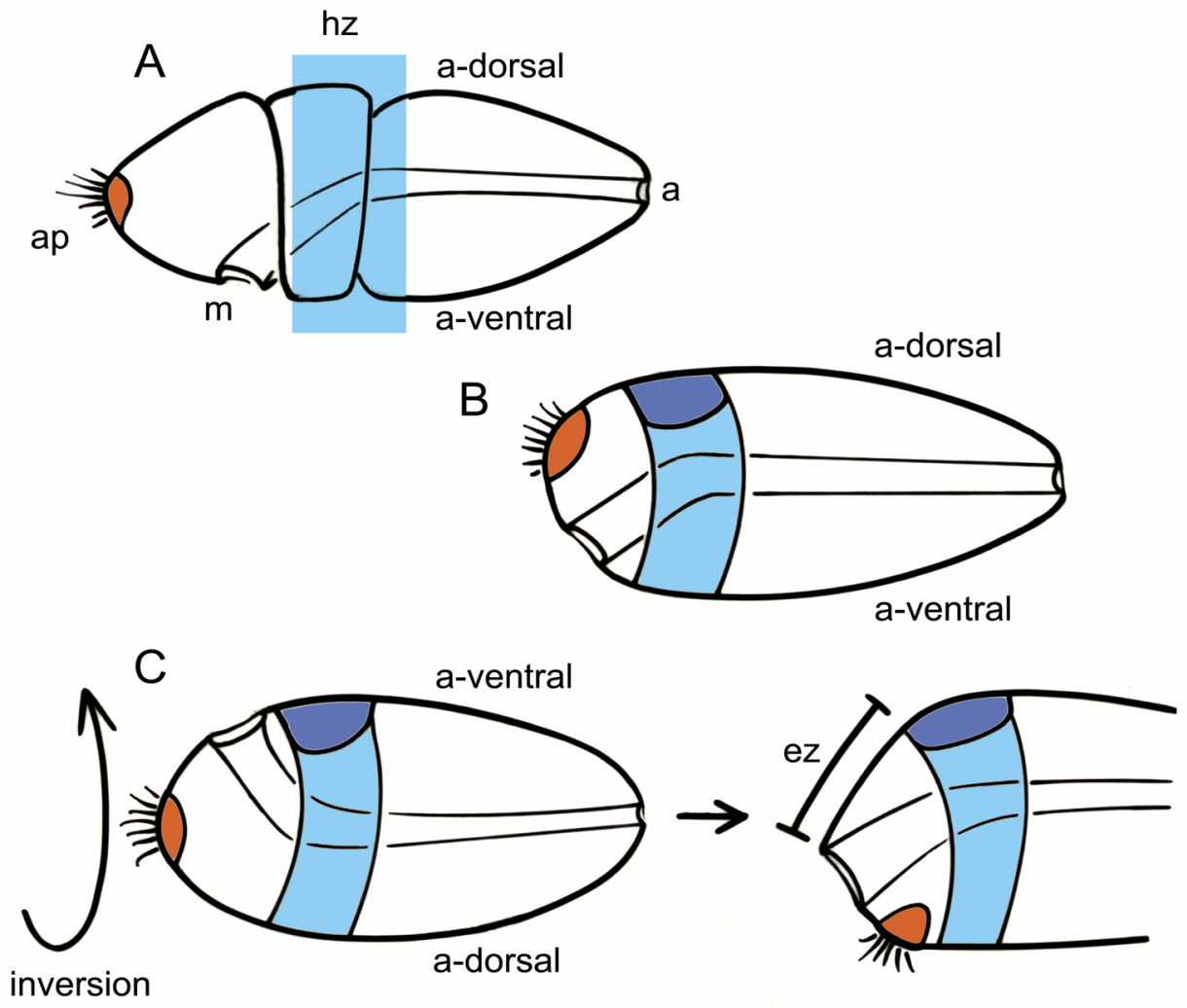

The ancestral chordate in this scenario, in its inverted position, would have both its mouth and brain on the dorsal surface of the body. While this may seem peculiar, it is exactly the situation in ascidian embryos and larvae [24]. What is then needed to generate the body of more advanced chordates like amphioxus and vertebrates is for differential growth of the ectoderm separating the mouth and brain to move the former forward, over the front or side of the snout, to what is now the ventral surface. The site of the ancestral apical organ must move as well, and will eventually find itself behind the now-ventral mouth, roughly in the position of the adhesive gland, which is also exactly the path the polar bodies follow from the apical pole of the egg during amphibian development [24]. In consequence, the anterior most part of our brain cannot be homologous with the anterior-most parts of protostome brains, because the latter are in part derived from apical structures. This is despite the supposed homology between the anterior otx- expressing regions of protostome and deuterostome brains, because in embryos and larvae in both groups, otx can be expressed both pre- and post-orally [25,26].

In summary, though there is good reason to doubt that the chordate neural tube actually derives evolutionarily from larval ciliary bands [19], Nielsen's point about the site of brain formation being postoral, though counterintuitive, is largely consistent with current molecular data. In addition, the larvae of hemichordates and echinoderms have neurons at this location, and these form comparatively complex ganglion-like assemblages in some instances. Various of the genes involved in neurogenesis are expressed here, including engrailed [27]. The function of these simple neural centers is to innervate the larval oral region and pharynx, presumably to control basic feeding behaviors and digestion. If these did indeed serve in some way as the core of the evolving chordate brain, then the progressive incorporation of centers for locomotory control into the brain is yet another story, and one that would have unfolded 
quite independently of the evolutionary process by which a centralized brain and nerve cord arose in the protostome lineage. In this version of events, it is quite unlikely that the internalized neural tube of chordates can be explained by a simple inversion of a protostome-like ancestor with an already-internalized brain and nerve cord, as has sometimes been supposed.

\section{Acknowledgments}

Supported by NSERC Canada. I thank Chris Lowe, Jean-Francois Brunet, Shunsuke Yaguchi and Linda Holland for access to unpublished data that, though not specifically mentioned, support the general line of argument made above.

\section{Conflict of Interest}

The author has declared that no conflict of interest exists.

\section{References}

1. Shimeld SM, Holland ND. Amphioxus molecular biology: insights into vertebrate evolution and developmental mechanisms. Can J Zool 2005; 83:90-100.

2. Mazet F, Shimeld SM. The evolution of chordate neural segmentation. Dev Biol 2002; 251:258-270.

3. Wicht $\mathrm{H}$, Lacalli TC. The nervous system of amphioxus: structure, development, and evolutionary significance. Can J Zool 2005; 83:122-150.

4. Lacalli TC, Kelly SJ. Ventral neurons in the anterior nerve cord of amphioxus larvae I. An inventory of cell types and synaptic patterns. J Morph 2003; 257:190-211.

5. Tallafuss A, Bally-Cuif L. Tracing of her5 progeny in zebrafish transgenics reveals the dynamics of midbrain-hindbrain neurogenesis and maintenance. Development 2003; 130:43074323.

6. Sato T, Joyner AL, Nakamura H. How does Fgf signaling from the isthmic organizer induce midbrain and cerebellum development? Dev Growth Diff 2004; 46:487-494.

7. Castro LFC, Kaltenbach SL, Holland PWH, Holland ND, Holland LZ. A Gbx homeobox gene in amphioxus: insights into ancestry of the ANTP class and evolution of the midbrain/hindbrain boundary. Dev Biol ; in press.

8. Holland LZ, Kene M, Williams N, Holland ND. Sequence and embryonic expression of the amphioxus engrailed gene (AmphiEn): the metameric pattern of transcription resembles that of its segment-polarity homolog in Drosophila. Development 1997; 124:1723-1732.

9. Kozmik Z, Holland ND, Kalousova A, Paces J, Schubert M, Holland LZ. Characterization of an amphioxus paired box gene, AmphiPax 2/5/8: developmental expression patterns in optic support cells, nephridium, thyroid-like structures and pharyngeal gill slits, but not in the midbrain-hindbrain boundary region. Development 1999; 126:1295-1304.

10. Bouchard M, Pfeffer P, Busslinger M. Functional equivalence of the transcriptional factors Pax2 and Pax5 in mouse development. Development 2000; 127:3703-3713.

11. Takahashi T, Holland PWH. Amphioxus and ascidian Dmbx homeobox genes give clues to the vertebrate origins of midbrain development. Development 2004; 131:3285-3294.

12. Wada H, Saiga H, Satoh N, Holland PWH. Tripartite organization of the ancestral chordate brain and the antiquity of placodes: insights from ascidian Pax-2/5/8, Hox and Otx genes. Development 1998; 125:1113-1122.
13. Canestro C, Bassham S, Postlethwait J. Development of the central nervous system in the larvacean Oikopleura dioica and the evolution of the chordate brain. Dev Biol 2005; 285:298-315.

14. Moret F, Guilland JC, Coudouel S, Rochette L, Vernier P. Distribution of tyrosine hydroxylase, dopamine and serotonin in the central nervous system of amphioxus: implications for the evolution of catecholamine systems in vertebrates. J Comp Neurol 2004; 468:135-150.

15. Candiani S, Oliveri D, Parodi M, Castagnola P, Pestarino M. AmphiD1/B, a D1/b-adrenergic receptor from the amphioxus Branchiostoma floridae: evolutionary aspects of the catecholaminergic system during development. Dev Gen Evol 2005; 215:631-638.

16. Lacalli TC, Holland LZ. The developing dorsal ganglion of the salp Thalia democratica, and the nature of the ancestral chordate brain. Phil Trans Roy Soc B 1998; 353:1943-1967.

17. Lacalli TC. New perspectives on the evolution of protochordate sensory and locomotory systems, and the origin of brains and heads. Phil Trans Roy Soc B 2001; 356:1565-1572.

18. Manni L, Agnoletto A, Zaniolo G, Burighel P. Stomodeal and neurohypophyseal placodes in Ciona intestinalis: insights into the origin of the pituitary gland. J Exp Zool 2005; 304B:324-339.

19. Lacalli TC. Protochordate body plan and the evolutionary role of larvae: old controversies resolved? Can J Zool 2005; 83:216-224.

20. Gerhart J, Lowe C. Kirschner M. Hemichordates and the origin of chordates. Curr Opin Gen Dev 2005; 15:461-467.

21. Lowe CJ, Wu M, Salic A, Evans L, Lander E, Strange-Thomann N, Gruber CE, Gerhart J, Kirschner M. Anteroposterior patterning in hemichordates and the origins of the chordate nervous system. Cell 2003; 113:853-865.

22. Ikuta $T$, Saiga H. Organization of Hox genes in ascidians: present, past, and future. Dev Dynam 2005; 232:382-389.

23. Reversade B, Kuroda H, Lee H, Mays A, DeRobertis EM. Depletion of Bmp2, Bmp4, Bmp7 and Spemann organizer signals induces massive brain formation in Xenopus embryos. Development 2005; 132:3381-3392.

24. Nielsen C. Origin of the chordate central nervous system - and the origin of chordates. Dev Gen Evol 1999; 209:198-205.

25. Tagawa K, Satoh N, Humphreys T. Molecular studies of hemichordate development: a key to understanding the evolution of bilateral animals and chordates. Evol Dev 2001; 3:443-454.

26. Arendt D, Technau U, Wittbrodt J. Evolution of the bilaterian larval foregut. Nature 2001; 409:81-85.

27. Yaguchi S, Nakajima Y, Wang D, Burke RD. Embryonic expression of engrailed in sea urchins. Gene Expr Patterns; in press. 